\title{
Psychosocial work conditions and registered sickness absence: a 3-year prospective cohort study among office employees
}

\author{
C. A. M. Roelen · P. C. Koopmans • \\ U. Bültmann · J. W. Groothoff · J. J. L. van der Klink
}

Received: 26 June 2008/ Accepted: 6 May 2009/Published online: 27 May 2009

(c) The Author(s) 2009. This article is published with open access at Springerlink.com

\begin{abstract}
Purpose To investigate associations between a wide variety of psychosocial work conditions and sickness absence in a medium-sized company.

Methods Prospective cohort study of 395 employees working in an insurance office. Self-reported psychosocial work conditions were measured by questionnaire in January 2002 and linked to registered sickness absence in the period January 2002 to December 2004 adjusting for earlier sick leave and psychological distress.

Results The questionnaires of 244 employees were eligible for analysis. Decision authority and co-worker support were associated with sickness absence days, but their associations with sickness absence episodes were not significant. Role clarity was associated with the number of sickness absence days, but only with the number of short sickness absence episodes in women.
\end{abstract}

C. A. M. Roelen

ArboNed Occupational Health Service, PO Box 158,

8000 AD Zwolle, The Netherlands

C. A. M. Roelen · P. C. Koopmans · U. Bültmann ·

J. W. Groothoff · J. J. L. van der Klink

Department of Health Sciences, University Medical Center,

Groningen, University of Groningen, PO Box 196,

9700 AD Groningen, The Netherlands

P. C. Koopmans

ArboNed Occupational Health Service, PO Box 141,

9700 AC Groningen, The Netherlands

C. A. M. Roelen ( $\square)$

ArboNed Corporate Accounts, PO Box 158, 8000 Zwolle,

The Netherlands

e-mail: corne.roelen@arboned.nl
Conclusions The wide variety of investigated psychosocial work conditions contributed little to the explanation of sickness absence in the medium-sized insurance office.

Keywords Psychosocial work conditions .

Sickness absence days $\cdot$ Sickness absence episodes

\section{Introduction}

New work practices and rapid technological advances are changing the nature of jobs. In many developed countries, unhealthy physical and chemical exposures in work have been substantially reduced, as well as their accompanying diseases. Work has become mentally demanding and there is a steady increase in workload leaving employees with less control over their work (Sparks et al. 2001; Smulders 2006). Employees are often being required to work beyond their contracted hours due to tight deadlines and understaffing. Moreover, many organisations are reducing their permanent workforce and converting to a culture of temporary contracts, increasing feelings of insecurity among the personnel (Parent-Thirion et al. 2007). These factors are associated with poor mental health and sickness absence (Stansfeld and Candy 2006).

Sickness absence is a strong predictor of disability and mortality (Kivimäki et al. 2003, 2004). Additionally, the economic costs for sickness absence are a substantial social burden in countries where employees receive sick-leave benefits. Although there is a large body of knowledge about the impact of the psychosocial work environment on the risk of sickness absence, the associations are still poorly understood (Allebeck and Mastekaasa 2004; Rugulies et al. 2007). Large-scale prospective studies, investigating demand-control-support variables, have found that low 
levels of control over work were related to high levels of sickness absence, whereas the results for demands and support were inconsistent (North et al. 1996; Niedhammer et al. 1998; Vahtera et al. 2000; Melchior et al. 2003; Moreau et al. 2004; Head et al. 2006). Psychological job demands are assumed to consist of different types of demands, such as amount of work, work pace and emotional demands (Kristensen et al. 2004). This might explain the inconclusive associations with sickness absence and calls for a more specific conceptualization of psychological demands (Rugulies et al. 2007). Moreover, other factors, such as job insecurity, role clarity, role conflict, the meaning of work, and fairness at work have recently been identified as predictors of sickness absence (Nielsen et al. 2004, 2006; Lund et al. 2005; Rugulies et al. 2007; Duijts et al. 2007). Thus, a more comprehensive approach is needed in which psychosocial work conditions are conceptualized broadly. In the present study, we investigated the prospective associations between a wide variety of psychosocial work conditions and sickness absence among office employees. Most studies on the associations between psychosocial work environment and sickness absence investigated large populations. It is necessary for occupational health practice to know whether the results of those large-scale studies suffice to characterize the psychosocial work environment of small- and medium-sized companies. Based on the literature, we hypothesize that job control in terms of decision latitude is also associated with sickness absence in a medium-sized insurance office employing 395 persons. Furthermore, we were interested in the question whether other psychosocial work determinants such as emotional demands, role clarity, role conflict, and job insecurity are associated with sickness absence in this company.

Earlier studies assessed sickness absence either by sick days or by episodes. In the present study, we measured both which enabled us to study differences in the associations of psychosocial work conditions with sickness absence days and sickness absence episodes.

\section{Method}

Study design and population

The present study is a prospective cohort study with a 3year follow-up of office employees, in which the questionnaire data are linked to sickness absence data registered by ArboNed Occupational Health Services. The study population was a sample of convenience and included the personnel, a medium-sized $(N=395)$ insurance company. Selection into the insurance office and into this particular work was similar in men and women. The employees had comparable educational levels (senior secondary vocational educations in administration, book-keeping or insurances), job grades, and income. All workers were office employees doing computer work. Employees in the insurance and public relations departments also had customer service tasks. Ethical approval was sought from the Medical Ethics Committee of the University Medical Center Groningen, who advised that ethical approval was not required.

\section{Measurement of psychosocial work conditions}

In January 2002, the insurance company distributed the Experience and Assessment of Work Questionnaire (Van Veldhoven and Meijman 1994) among the personnel and asked them to return the completed questionnaire by post to ArboNed Occupational Health Services. The self-administered questionnaire consisted of 27 subscales comprising a total of 232 questions on work conditions, which were answered on a four-point Likert-scale ranging from 0 (=always) to 3 (=never). The internal consistency of the subscales was characterized by rho $(\rho)$ and scales with $\rho>0.80$ were considered consistent (Drenth and Sijtsma 1990). For this study, we used 12 subscales: work pace (11 items; $\rho=0.89$ ), emotional demands ( 7 items; $\rho=0.85$ ), psychological workload (11 items; $\rho=0.87$ ), repetitive work (6 items; $\rho=0.82$ ), educational opportunities (4 items; $\rho=0.84$ ), job autonomy (11 items, $\rho=0.90)$, decision authority ( 8 items; $\rho=0.85$ ), supervisor support (9 items; $\rho=0.90)$, co-worker support $(9$ items; $\rho=0.87$ ), role clarity (9 items; $\rho=0.81$ ), role conflict ( 9 items; $\rho=0.80$ ), and job insecurity (4 items; $\rho=0.95$ ). The scores of each subscale were standardized according to the formula:

\section{$\frac{\text { subscale score }}{3 \times \text { number of subscale items }} \times 100$}

after which all subscales obtained a score between 0 and 100 .

The subscales job autonomy, decision authority, supervisor support, and co-worker support were reversed, meaning that high scores corresponded with low autonomy, low decision authority, and low support, respectively.

The Experience and Assessment of Work Questionnaire also measured the need for recovery after work (11 yes/no items about fitness and relaxation after work; $\rho=0.87$ ), rumination (4 yes/no items about worrying; $\rho=0.80$ ), emotional reactions (12 yes/no items; $\rho=0.89$ ), and sleep problems ( 14 yes/no items; $\rho=0.95$ ). The scores of these scales were added up and according to Van Veldhoven and Meijman Th (1994) reflected psychological distress, which we regarded as a proxy for the mental health status of the employees with higher scores representing more distress and poorer mental health. 
Measurement of sickness absence

The questionnaire data were linked to prospective sickness absence data retrieved from the records of ArboNed Occupational Health Services in which the first and last dates of all absences were registered. Sickness absence days and episodes were calculated on the individual level. We counted the total number of sickness absence days for each employee in the period January 1st 2002 to December 31 st 2004, irrespective of contracted hours and considering partial days off as full sick days. Employees who were sick-listed in January 1st 2002 were excluded from the analysis. Sickness absence days were counted until December 31st 2004, even if the employee remained on sick-leave thereafter. The number of sickness absence episodes between January 1st 2002 and December 31st 2004 was also counted for each employee, distinguishing between short episodes (1-21 days) of uncertified absence, and long episodes ( $>21$ days) of mostly certified sickness absence. Earlier, the sick-leave was assessed on the individual level by the total number of sickness absence days in the period January 2000 through December 2001.

\section{Statistical analyses}

The number of absence days was skewed to the right [mean 48.9 days, standard deviation (SD) 82.8 days; median 18.0 days]. Normal distribution was approximated after logarithmic transformation: mean $2.9(\mathrm{SD}=1.5)$ and median 2.9. The prospective associations between psychosocial work conditions and the log-transformed number of sickness absence days were analyzed with multiple linear regression (SPSS for Windows, version 15) controlling for earlier sick-leave and psychological distress. The linear regression models fitted the number of sickness absence days in men and women well but explained little $(12-14 \%)$ of the variance in the number of sickness absence days.

To examine the prospective associations between psychosocial work conditions and sickness absence episodes, a Poisson regression model was computed using GENLOG for general log-linear analysis in SPSS for Windows version 15. The Poisson distribution implies that the variance is equal to the mean $(\mu)$. The Poisson model showed a good fit for the number of long episodes. The variance in the number of short episodes of absence, however, was greater than the mean resulting in overdispersion. Therefore, a zero-inflated negative binomial distribution was estimated for short absences using Transition Data Analysis version 6.4f (Blossfeld and Rohwer 2002). The negative binomial distribution proved to be a better fit for the number of short sickness absence episodes. In the negative binomial model and the Poisson regression model earlier sick-leave and psychological distress were adjusted for.

\section{Results}

Of the distributed 395 questionnaires, 265 (67\%) were returned to the occupational health service. Twenty-one questionnaires were excluded because they were not complete. Thus, a total of 151 employees (64 men and 87 women) were not eligible for analysis. These non-participants were 39.2 [standard deviation $(\mathrm{SD})=7.1$ ] years of age, had 6,271 sickness absence days and a total of 732 sickness absence episodes, of which 686 short episodes and 46 long episodes, during follow-up. 244 participants (103 men and 141 women) were $39.0(\mathrm{SD}=8.9)$ years of age which did not differ significantly from non-participants (Mann-Whitney $U$ test: $P=0.977$ ). They had 11,940 sickness absence days, and a total of 1,085 sickness absence episodes, 991 short episodes and 94 long episodes which did not differ significantly from non-participants considering Mann-Whitney $U$ test results $P=0.465$ (for short episodes) and $P=0.338$ (for long episodes). None of the participants dropped out during the 3-year study period. The sickness absence characteristics of participating men and women are summarized in Table 1. Men had 350 sickness absence episodes with a mean duration of $10.0(\mathrm{SD}=31.1)$ days and women had 735 sickness absence episodes with a mean duration of $13.3(\mathrm{SD}=39.3)$ days.

Table 1 Descriptive statistics of sickness absence parameters

\begin{tabular}{|c|c|c|c|c|c|c|c|c|c|c|c|c|}
\hline & \multicolumn{4}{|c|}{ Total $(N=244)$} & \multicolumn{4}{|c|}{$\operatorname{Men}(N=103)$} & \multicolumn{4}{|c|}{ Women $(N=141)$} \\
\hline & $N$ & Mean & SD & Median & $N$ & Mean & SD & Median & $N$ & Mean & SD & Median \\
\hline Total episodes & 1,085 & 4.4 & 3.8 & 4 & 350 & 3.4 & 2.8 & 3 & 735 & 5.2 & 4.2 & 5 \\
\hline Short (1-21 days)episodes & 991 & 4.1 & 3.5 & 3 & 327 & 3.2 & 2.7 & 2 & 664 & 4.7 & 3.9 & 4 \\
\hline Long ( $>21$ days) episodes & 94 & 0.4 & 0.7 & 0 & 23 & 0.2 & 0.5 & 0 & 71 & 0.5 & 0.8 & 0 \\
\hline Sick days during study (from 2002 to 2004) & 11,940 & 48.9 & 82.8 & 18 & 3,304 & 32.3 & 62.2 & 12 & 8,636 & 61.1 & 93.4 & 26 \\
\hline Earlier sick days (in 2000 and 2001) & 4,566 & 18.7 & 51.3 & 3 & 976 & 9.4 & 32.6 & 3 & 3,590 & 25.4 & 60.7 & 4 \\
\hline
\end{tabular}

$S D$ standard deviation, $S E$ standard error of mean 
Table 2 Associations between psychosocial work conditions and the number of sickness absence days

\begin{tabular}{|c|c|c|c|c|c|c|}
\hline \multirow{2}{*}{$\begin{array}{l}\text { Psychosocial work } \\
\text { condition (Reference) }\end{array}$} & \multicolumn{2}{|c|}{ Total $(N=244)$} & \multicolumn{2}{|c|}{$\operatorname{Men}(N=103)$} & \multicolumn{2}{|c|}{ Women $(N=141)$} \\
\hline & Mean (SD) & $b(\mathrm{SE})$ & Mean (SD) & $b(\mathrm{SE})$ & mean $(\mathrm{SD})$ & $b \quad(\mathrm{SE})$ \\
\hline Gender & & $-0.44(0.21)^{*}$ & & & & \\
\hline Age & $39.0(8.9)$ & $0.01(0.01)$ & $40.3(8.9)$ & $0.00(0.02)$ & $38.0(8.8)$ & $0.02(0.02)$ \\
\hline Work pace (42) & $41(15)$ & $0.03(0.08)$ & $42(12)$ & $0.20(0.14)$ & $41(16)$ & $-0.05(0.10)$ \\
\hline Emotional demands (25) & $27(12)$ & $0.05(0.10)$ & $27(11)$ & $0.06(0.16)$ & $27(13)$ & $0.05(0.13)$ \\
\hline Psychological workload (74) & $76(16)$ & $0.04(0.08)$ & $75(15)$ & $-0.07(0.12)$ & $77(17)$ & $0.08(0.10)$ \\
\hline Repetitive work (44) & $43(21)$ & $0.08(0.08)$ & $37(20)$ & $0.02(0.11)$ & $48(20)$ & $0.10(0.11)$ \\
\hline Educational opportunities (53) & $51(20)$ & $-0.05(0.07)$ & 49 (19) & $-0.10(0.12)$ & $52(21)$ & $-0.04(0.10)$ \\
\hline Job autonomy $(39)^{\mathrm{a}}$ & $41(20)$ & $-0.02(0.06)$ & $35(17)$ & $-0.01(0.11)$ & $45(21)$ & $-0.06(0.08)$ \\
\hline Decision authority $(52)^{\mathrm{a}}$ & $46(19)$ & $0.18(0.08)^{*}$ & $41(20)$ & $0.26(0.13)^{\#}$ & $49(17)$ & $0.17(0.12)$ \\
\hline Supervisor support $(22)^{a}$ & $19(13)$ & $0.02(0.10)$ & $19(12)$ & $0.09(0.17)$ & $18(15)$ & $0.06(0.13)$ \\
\hline Co-worker support $(21)^{\mathrm{a}}$ & $21(11)$ & $0.22(0.10)^{*}$ & $22(11)$ & $0.16(0.17)$ & $21(12)$ & $0.22(0.14)$ \\
\hline Role clarity (34) & $28(15)$ & $-0.17(0.08)^{*}$ & $29(14)$ & $-0.07(0.13)$ & $27(15)$ & $-0.25(0.11)^{*}$ \\
\hline Role conflict (20) & $17(11)$ & $-0.05(0.11)$ & $17(11)$ & $0.02(0.16)$ & $17(11)$ & $-0.09(0.15)$ \\
\hline Job insecurity (46) & $28(31)$ & $0.00(0.04)$ & $27(30)$ & $-0.11(0.06)^{\#}$ & $23 \pm 28$ & $0.06(0.05)$ \\
\hline$R^{2}$ & & 0.124 & & 0.141 & & 0.137 \\
\hline Post-hoc statistical power & & 0.978 & & 0.671 & & 0.838 \\
\hline
\end{tabular}

${ }^{a}$ Reversed scales, meaning that high scale scores represent low levels of the work condition

\# $P<0.10, * P<0.05$

Mean and standard deviation (SD) of the psychosocial work conditions on a score range of 0 (low) to 100 (high), with exception of the scales job autonomy, decision latitude, supervisor support and co-worker support which had reversed scores $(0=$ high and $100=$ low $)$. The table also shows the reference scores for the Dutch financial sector. The results of multiple linear regression analysis using the model $\ln (y)=a+b_{1} x_{1}+b_{2} x_{2}+\ldots . .+b_{\mathrm{i}} x_{\mathrm{i}}$ are presented in regression coefficients (b) with their standard errors (SE) adjusted for earlier sick-leave and psychological distress. The $R^{2}$ value is a measure of the proportion of explained variety in sickness absence days

Psychosocial work conditions and sickness absence days

Men had lower scores on repetitive work than women as shown in Table 2, with $P<0.01$ using the Mann-Whitney $U$ test. Men also had lower scores on job autonomy $(P<0.01)$ and decision authority $(P<0.01)$, meaning they perceived higher autonomy and decision authority in their work than women.

The associations between the psychosocial work conditions and sickness absence days are also presented in Table 2. The total population decision authority $(P=0.04)$ and co-worker support $(P=0.03)$ were positively related to the number of sickness absence days. Because these scales had reversed scores, this meant that the higher decision authority and higher co-worker support were associated with fewer sickness absence days. Role clarity was negatively related $(P=0.04)$ to the number of sickness absence days. Gender was significantly associated with the number of sickness absence days; therefore we stratified the results by gender. In men, the decision authority was associated with the number of sickness absence days, though marginally significant $(P=0.05)$. Job insecurity was non-significantly associated $(P=0.06)$ with the number of absence days in men. In women, the role clarity was negatively associated $(P=0.03)$ with the number of sickness absence days during follow-up.

Psychosocial work conditions and sickness absence episodes

Table 3 shows the associations between psychosocial work conditions, and the number of short and long episodes of sickness absence. We found significant gender differences and the number of long sickness absence episodes were higher with increasing age [rate ratio $(\mathrm{RR})=1.38$; $P=0.02]$. Therefore, we chose to stratify the results by gender and adjust for age in the analyses.

In men, the highest RR was found for co-worker support with regard to short episodes of sickness and for work pace when long episodes were considered. However, the associations between these work conditions and the number of sickness absence episodes were not statistically significant (Table 3). In women, the work pace $(\mathrm{RR}=0.89, P=0.02)$ and role clarity $(\mathrm{RR}=0.86$, $P=0.01)$ were negatively related to the number of short episodes of sickness absence. When long episodes were 
Table 3 Associations between psychosocial work conditions and the number of sickness absence episodes

\begin{tabular}{|c|c|c|c|c|c|c|}
\hline \multirow[t]{2}{*}{ Psychosocial work condition } & \multicolumn{2}{|l|}{ Total population } & \multicolumn{2}{|l|}{ Men } & \multicolumn{2}{|l|}{ Women } \\
\hline & $\begin{array}{l}\text { Short episodes } \\
\text { RR }(95 \% \text { CI) }\end{array}$ & $\begin{array}{l}\text { Long episodes } \\
\text { RR }(95 \% \text { CI) }\end{array}$ & $\begin{array}{l}\text { Short episodes } \\
\text { RR (95\% CI) }\end{array}$ & $\begin{array}{l}\text { Long episodes } \\
\text { RR }(95 \% \text { CI) }\end{array}$ & $\begin{array}{l}\text { Short episodes } \\
\text { RR }(95 \% \text { CI })\end{array}$ & $\begin{array}{l}\text { Long episodes } \\
\text { RR }(95 \% \mathrm{CI})\end{array}$ \\
\hline Gender & $0.83(0.66-1.05)$ & $0.50(0.30-0.85)^{*}$ & & & & \\
\hline Age & $0.87(0.76-1.00)^{\#}$ & $1.38(1.05-1.82)^{*}$ & $0.81(0.63-1.03)$ & $1.44(0.73-2.78)$ & $0.93(0.78-1.10)$ & $1.46(1.06-2.01)^{*}$ \\
\hline Work pace & $0.93(0.85-1.01)^{\#}$ & $1.04(0.89-1.21)$ & $0.97(0.81-1.16)$ & $1.31(0.83-2.08)$ & $0.89(0.81-0.98)^{*}$ & $0.97(0.80-1.18)$ \\
\hline Emotional demands & $0.94(0.85-1.05)$ & $1.18(0.96-1.44)$ & $0.99(0.82-1.21)$ & $0.93(0.55-1.57)$ & $0.94(0.83-1.06)$ & $1.17(0.93-1.49)$ \\
\hline Psychological workload & $1.03(0.95-1.12)$ & $0.90(0.76-1.06)$ & $0.90(0.79-1.04)$ & $0.94(0.65-1.34)$ & $1.09(0.98-1.22)$ & $0.87(0.70-1.07)$ \\
\hline Repetitive work & $1.01(0.93-1.10)$ & $1.08(0.91-1.28)$ & $0.96(0.84-1.10)$ & $1.19(0.83-1.69)$ & $1.03(0.93-1.15)$ & $1.05(0.85-1.30)$ \\
\hline Educational opportunities & $0.96(0.89-1.04)$ & $0.94(0.80-1.10)$ & $0.95(0.81-1.10)$ & $0.98(0.68-1.42)$ & $0.97(0.88-1.06)$ & $0.93(0.77-1.12)$ \\
\hline Job autonomy $^{\mathrm{a}}$ & $1.03(0.96-1.11)$ & $0.97(0.85-1.11)$ & $1.07(0.94-1.21)$ & $0.96(0.69-1.34)$ & $1.00(0.92-1.09)$ & $1.01(0.86-1.18)$ \\
\hline Decision authority $^{\mathrm{a}}$ & $1.01(0.92-1.10)$ & $1.18(0.98-1.42)^{\#}$ & $1.04(0.90-1.22)$ & $1.23(0.81-1.88)$ & $1.02(0.90-1.14)$ & $1.10(0.89-1.37)$ \\
\hline Supervisor support ${ }^{\mathrm{a}}$ & $1.05(0.95-1.16)$ & $0.97(0.79-1.18)$ & $0.91(0.74-1.12)$ & $1.08(0.64-1.81)$ & $1.08(0.95-1.24)$ & $0.98(0.77-1.23)$ \\
\hline Co-worker support ${ }^{\mathrm{a}}$ & $1.09(0.97-1.21)$ & $1.21(0.96-1.51)$ & $1.13(0.93-1.38)$ & $1.23(0.79-2.07)$ & $1.12(0.99-1.26)$ & $1.14(0.87-1.50)$ \\
\hline Role clarity & $0.92(0.84-1.01)^{\#}$ & $0.87(0.73-1.05)$ & $0.99(0.86-1.14)$ & $0.82(0.54-1.27)$ & $0.86(0.76-0.97)^{*}$ & $0.88(0.70-1.09)$ \\
\hline Role conflict & $0.99(0.88-1.10)$ & $0.83(0.66-1.05)$ & $1.04(0.87-1.25)$ & $1.08(0.65-1.79)$ & $0.95(0.82-1.09)$ & $0.79(0.59-1.06)$ \\
\hline Job insecurity & $1.00(0.96-1.04)$ & $0.96(0.88-1.04)$ & $0.95(0.89-1.02)$ & $0.90(0.75-1.08)$ & $1.03(0.98-1.08)$ & $0.95(0.86-1.04)$ \\
\hline
\end{tabular}

${ }^{a}$ Reversed scales, meaning that high scale scores represent low levels of the work condition

\# $P<0.10, * P<0.05$

The table presents the rate ratios (RR), adjusted for earlier sick-leave and psychological distress, and their 95\% confidence intervals (95\% CI) for the associations between the total number of sickness absence episodes, short (1-21 days) sickness absence episodes and long ( $>21$ days) sickness absence episodes. The rate ratios show the effect of a 10-point increase on the psychosocial scales

considered, the highest RR was found for emotional demands and co-worker support, but these associations were not significant.

\section{Discussion}

In this study, we prospectively analyzed associations of a wide range of psychosocial work conditions in a mediumsized insurance company with the number of registered sickness absence days and episodes in both genders, adjusting for earlier sick-leave and psychological distress, the latter being regarded as a proxy for the mental health status. The associations between psychosocial work conditions and sickness absence days differed from those between psychosocial work conditions and episodes of sickness absence. Decision authority and co-worker support were significantly associated with the number of sickness absence days but not episodes. Furthermore, the associations between psychosocial work conditions and sickness absence were gender-specific. Decision authority was associated with the number of sickness absence days in men but not in women. Role clarity was associated with the number of sickness absence days in women but not in men. Role clarity was also associated with the number of short episodes of sickness absence in women but not in men.
In most studies, the sickness absence is determined by counting the episodes of absence which are often divided into short and long episodes. North et al. (1996) examined the association between the psychosocial work environment and subsequent rates of short ( $\leq 7$ days) and long ( $>7$ days) episodes of absence in 10,314 British civil servants. They found the levels of control, in terms of variety and use of skills, and support at work to predict the rates of short and, to a lesser extent, long episodes of absence. The GAZEL cohort studies included 12,555 employees working in the French national electricity and gas company, and showed that low levels of decision latitude for both sexes and low job support for males were significant predictors of the number of sickness absence episodes (Niedhammer et al. 1998; Melchior et al. 2003). Associations of job demands, decision latitude, and support at the workplace with the number of sickness absence episodes, however, could not be confirmed in our study among the personnel of a medium-sized company. Our study population was smaller and probably the results were dispersed by individual variations in coping with work conditions. Secondly, as all participants were officers working in the same company there was little variation in job content, work conditions, and organizational culture. Finally, the personnel of a company interact with each other, from which the question arises whether they can be considered independent. Christensen et al. (2005) studied sickness absence 
at the company level and found different associations between the psychosocial work conditions and sickness absence in different companies.

Nielsen et al. (2006) investigated a broad variety of psychosocial work conditions in a population of 1,919 employees working in the private and public sector. They found a positive association between skill discretion and the number of short episodes of sickness absence in women. Among men, the short episodes were associated with the meaning of work. As for long episodes of sickness absence, the associations were reported for psychological work demands and decision authority in women, and both decision authority and supervisor support in men. Our results confirmed that the associations were gender-specific for role clarity being related to the number of short episodes of sickness absence in women but not in men. However, the associations between psychosocial work conditions and long episodes of sickness absence were neither found in men nor found in women. It should be noted that Nielsen et al. (2006) considered absences lasting more than 10 days as long episodes while we defined absences of more than 21 days as long in line with the GAZEL cohort studies. Differences in the definition of the duration of sickness absence episodes are a common shortcoming in sickness absence research, hindering the comparability of results.

Another way to measure sickness absence is to count the number of sickness absence days during follow-up. Rugulies et al. (2007) found client-specific demands (violence and threats from clients, emotional demands, and demands for hiding emotions), influence at work, the meaning of work, the quality of management, and role conflicts to be related to the number of sickness absence days in 890 human service workers. They used selfreported sickness absence data, asking workers for the number of sickness absence days in the last 12 months. We used recorded prospective sickness absence data, which were free of recall-bias, and found that high decision authority was associated with fewer sickness absence days. Role clarity was negatively related to the number of sickness absence days. Emotional demands were not related to the number of registered sickness absence days. Personal client contacts are probably more common in the human service sector than in the insurance sector where most client contacts are by telephone.

\section{Strengths and limitations of the study}

The strength of the study is that we used registered sickness absence data instead of self-reported sickness absence. Moreover, there was no loss to follow-up in the 3-year study period. Sickness absence as outcome variable was followedup after baseline measurement of psychosocial work conditions in January 2002, thereby limiting shared method variance or shared response biases. Earlier sick-leave and psychological distress, a proxy for the mental health status, were controlled for all statistical analyses. However, the information about factors not related to the workplace but known to influence sickness absence, such as marital state, number of children, leisure time activities, lifestyle, and social support outside work was not available. Another limitation was the fact that psychosocial work conditions were assessed at baseline only. Changes in perceptions cannot be ruled out, although there were no organizational changes in terms of reorganization, merge, managerial changes, or changes in work schedules or activities during follow-up. Finally, the results are at the most representative for office employees belonging to the upper-modal income levels.

In conclusion, the prospective associations between psychosocial work conditions and the number of sickness absence days differed from those between psychosocial work conditions and the number of sickness absence episodes. Decision latitude was significantly associated with the number of sickness absence days but not episodes. Thus, our hypothesis that decision latitude is associated with sickness absence was only partly confirmed. Role clarity was associated with the number of sickness absence days but only with the number of short sickness absence episodes in women. Other results were also gender-specific without a clear pattern for both genders. The investigated psychosocial work conditions explained $12-14 \%$ of the variance in sickness absence days. This suggests that other factors might be more important determinants of sickness absence in the investigated medium-sized insurance office.

Moreover, our results show that relying on the usual work determinants such as job demands, job control, and job support would be insufficient to characterize the psychosocial work environment of small- and medium-sized companies. Determinants of the psychosocial work environment should be assessed more broadly to develop tailormade company-specific interventions aimed at improving psychosocial work conditions.

Conflict of interest The authors declare that they have no conflict of interest.

Open Access This article is distributed under the terms of the Creative Commons Attribution Noncommercial License which permits any noncommercial use, distribution, and reproduction in any medium, provided the original author(s) and source are credited.

\section{References}

Allebeck P, Mastekaasa A (2004) Swedish Council on Technology Assessment in Health Care (SBU), chapter 5. Risk factors for sick leave-general studies. Scand J Public Health Suppl 63:49-108 
Blossfeld HP, Rohwer G (2002) Techniques of event history modelling. New approaches to causal analysis. Lawrence Erlbaum Associates Inc, Mahwah

Christensen KB, Nielsen ML, Rugulies R, Smith-Hansen L, Kristensen TS (2005) Workplace levels of psychosocial factors as prospective predictors of registered sickness absence. J Occup Environ Med 47:933-940

Drenth JD, Sijtsma K (1990) Introduction into the theory of psychological tests and their applications. Bohn, Stafleu \& Van Loghum, Houten

Duijts SF, Kant IJ, Swaen GM, van den Brandt PA, Zeegers MP (2007) A meta-analysis of observational studies identifies predictors of sickness absence. J Clin Epidemiol 60:1105-1115

Head J, Kivimäki M, Martikainen P, Vahtera J, Ferrie JE, Marmot MG (2006) Influence of change in psychosocial work characteristics on sickness absence: the Whitehall II study. J Epidemiol Community Health 60:55-61

Kivimäki M, Head J, Ferrie JE, Shipley MJ, Vahtera J, Marmot MG (2003) Sickness absence as a global measure of health: evidence from mortality in the Whitehall II prospective cohort study. BMJ 327:364

Kivimäki M, Forma P, Wikstrom J, Halmeenmaki T, Pentti J, Elovaino M et al (2004) Sickness absence as a risk marker of future disability pension: the 10-town study. J Epidemiol Community Health 58:710-711

Kristensen TS, Bjorner JB, Christensen KB, Borg V (2004) The distinction between work pace and working hours in the measurement of quantitative demands. Work \& stress 18:305322

Lund T, Labriola M, Christensen KB, Bültmann U, Villadsen E, Burr $\mathrm{H}$ (2005) Psychosocial work environment exposures as risk factors for long-term sickness absence among Danish employees: results from the DWECS/DREAM. J Occup Environ Med 47:1141-1147

Melchior M, Niedhammer I, Berkman LF, Goldberg M (2003) Do psychosocial work factors and social relations exert independent effects on sickness absence? A six-year prospective study of the GAZEL cohort. J Epidemiol Community Health 57:285-293

Moreau M, Valente F, Mak R et al (2004) Occupational stress and the incidence of sick leave in the Belgian workforce: the Belstress study. J Epidemiol Community Health 58:507-516
Niedhammer I, Bugel I, Goldberg M, Leclerc A, Guéguen A (1998) Psychosocial factors at work and sickness absence in the Gazel cohort: a prospective study. Occup Environ Med 55:735-741

Nielsen ML, Rugulies R, Christensen KB, Smith-Hansen L, Bjorner JB, Kirstensen TS (2004) Impact of the psychosocial work environment on registered absence from work: a two-year longitudinal study using the IPAW cohort. Work \& Stress 18:323-335

Nielsen ML, Rugulier R, Christensen KB, Smith-Hansen L, Kristensen TS (2006) Psychosocial work environment predictors of short and long spells of registered sickness absence during a 2year follow up. J Occup Environ Med 48:591-598

North F, Syme SL, Feeney A, Shipley M, Marmot M (1996) Psychosocial work environment and sickness absence among British civil servants: the Whitehall II Study. Am J Public Health 86:332-340

Parent-Thirion A, Macías FE, Hurley J, Vermeylen G (2007) Fourth European working conditions survey. European Foundation for the Improvement of Living and Working Conditions. Dublin, Ireland

Rugulies R, Christensen KB, Borritz M, Villadsen E, Bültmann U, Kristensen TS (2007) The contribution of the psychosocial work environment to sickness absence in human service workers: results of a 3-year follow-up study. Work \& Stress 21:293-311

Smulders PGW (2006) Worklife in the Netherlands. TNO Quality of Life, Hoofddorp

Sparks K, Faragher B, Cooper CL (2001) Well-being and occupational health in the 21st century workplace. J Occup Organ Psych 74:489-509

Stansfeld S, Candy B (2006) Psychosocial work environment and mental health - a meta-analytic review. Scand J Work Environ Health 32:443-462

Vahtera J, Kivimäki M, Pentti J, Theorell T (2000) Effect of change in the psychosocial work environment on sickness absence: a seven year follow up of initially health employees. J Epidemiol Community Health 54:484-493

Van Veldhoven M, Meijman Th F (1994) The measurement of psychological job demands with a questionnaire: the Experience and Assessment of Work Questionnaire. NIA, Amsterdam 